\title{
Transatlantica
}

Revue d'études américaines. American Studies Journal

\section{Entretien avec Dolores Marat}

Avignon, le 24 août 2013

\section{Muriel Adrien}

\section{(2) OpenEdition}

Journals

Édition électronique

URL : https://journals.openedition.org/transatlantica/6760

DOI : 10.4000/transatlantica.6760

ISSN : 1765-2766

Éditeur

Association française d'Etudes Américaines (AFEA)

Édition imprimée

Date de publication : 31 décembre 2013

Référence électronique

Muriel Adrien, «Entretien avec Dolores Marat », Transatlantica [En ligne], 2 | 2013, mis en ligne le 30 septembre 2014, consulté le 02 février 2023. URL : http://journals.openedition.org/transatlantica/6760 ; DOI : https://doi.org/10.4000/transatlantica.6760

Ce document a été généré automatiquement le 2 février 2023

\section{cc) (i) (2)}

Creative Commons - Attribution - Pas d'Utilisation Commerciale - Pas de Modification 4.0 International - CC BY-NC-ND 4.0

https://creativecommons.org/licenses/by-nc-nd/4.0/ 


\title{
Entretien avec Dolores Marat
}

\author{
Avignon, le 24 août 2013
}

\section{Muriel Adrien}

\section{NOTE DE L'AUTEUR}

J'ai rencontré Dolores Marat le 24 août 2013 chez elle en Avignon. Sa parole a été très libre et généreuse. N'étant pas autorisée à rendre publics les événements les plus intimes qu'elle m'a relatés, j'ai choisi ici de retranscrire une version expurgée de cet entretien. En revanche, je n'ai pas modifié ses réponses, comme en témoignent les nombreuses traces d'oralité.

Muriel Adrien remercie chaleureusement l'artiste pour la générosité qu'elle a eue à lui accorder un entretien, qui plus est chez elle, et à lui donner l'autorisation de reproduire les photographies.

Dolores Marat est née en 1944. Elle a exposé à différents endroits en France (dont Paris, Toulouse et Arles, en Corse, à St Barthélémy), en Angleterre (Londres, Liverpool), en Belgique (Bruxelles), au Portugal, au Pays-Bas, en Indonésie, aux États-Unis (New York). Ses photographies figurent dans des collections telles que le Musée de la photographie, Charleroi (Belgique), Galerie Frédéric Bazille, Montpellier (F), Fnac (F), Artothèque de Nantes (F), L'œil Écoute, Solignac (F), Coimbra (Portugal), MEP, NSM vie (F), Fond National pour l'Art Contemporain, Cabinet des Estampes, Bibliothèque Nationale de France. Parmi les dizaines de livres de Dolores Marat, l'un d'entre eux porte sur New York (New York USA, Marval, 2002). Son travail d'auteur utilise uniquement le procédé de tirage Fresson. 


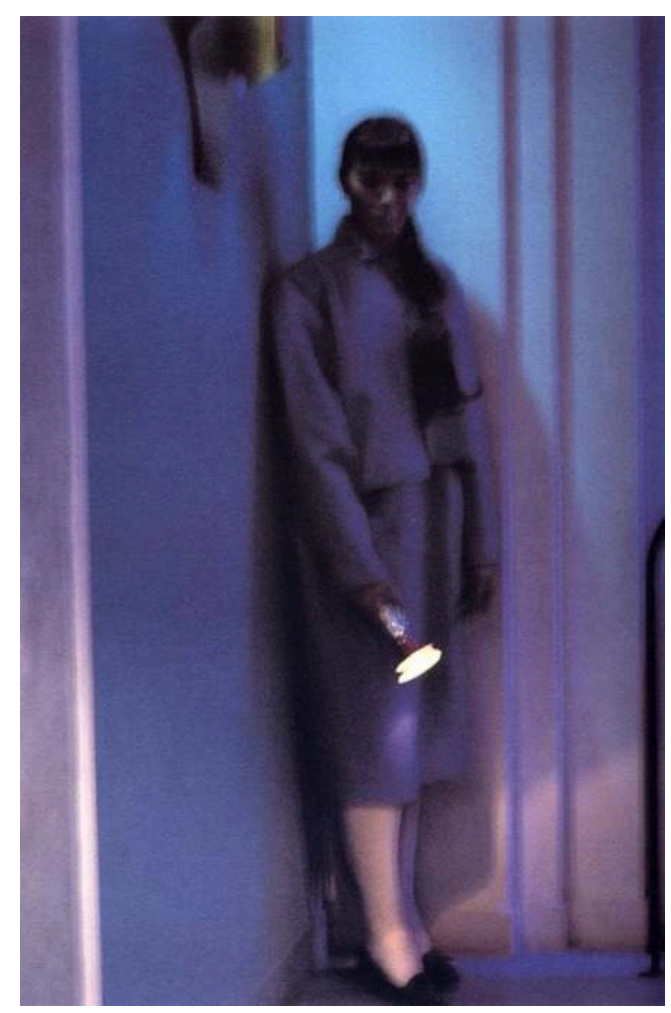

Paris, Sans titre [n.d]

\section{Muriel Adrien : Comment êtes-vous venue à la photographie?}

Dolores Marat : J'ai travaillé très tôt, à 15 ans. J'étais culottière-giletière, je faisais des pantalons. À un moment, j'ai eu 15 jours de vacances. Ma mère va faire ses courses dans un petit village à côté, à 5 kilomètres, en vélo. Elle revient, et elle me dit : « Tu sais, Froissard (c'était le photographe qui avait une petite boutique où il vendait des appareils photo, et où il développait des travaux d'amateurs), il cherche une bonne à tout faire. " J'ai dit: "J'y vais ». Avant, j'avais dit à ma mère que je voulais être photographe, et elle m'avait répondu : « Non ma fille, tu ne seras pas photographe, tu seras couturière ». Ma mère était paysanne. Quand il n'y avait aucune culture, comme chez moi, pas d'argent, les filles étaient dans la couture et les garçons dans la mécanique. Il fallait travailler, c'était après la guerre, c'était en fait avant les années 60. Je lui ai dit, du haut de mes 15 ans: «Tu ne peux pas m'empêcher de travailler pendant les vacances, j'y vais. » Donc je vais voir le monsieur avec un sourire jusquelà, je lui ai dit : «Je viens pour l'annonce ». Il me dit : « Bon d'accord, tu vois le balai, voilà, tout est là. » J'ai fait le ménage. J'étais heureuse comme tout. Il m'a gardée tout de suite. Comme j'étais venue en vélo, je pouvais repartir le soir, tranquille. Le vendredi, il me dit : « Dis donc, toi, combien tu gagnes, à faire tes pantalons? » C'était très peu. Il rajoute, sans rien me dire d'autre : «Si tu veux, ce soir, je te ramène chez toi, on met ton vélo dans la voiture, et je parle à ta mère. » Alors là, j'avais la banane jusque-là. Effectivement, il me ramène, et il dit à ma mère : «Écoutez madame, je crois que votre fille est douée pour la photo; si vous êtes d'accord, je vous offre tant en plus - je donnais tout à ma mère - et je la garde en apprentissage. » Et comme cela venait de quelqu'un qu'elle connaissait - parce que de temps en temps, elle lui donnait une bobine à développer - elle a dit : «D'accord!» Bien sûr, je faisais le ménage, mais à midi, j'avais fini, donc il m'expliquait comment on développait, comment on tirait, comment on faisait la retouche. Et c'est vrai, alors que j'y 
connaissais rien, mais rien du tout, pour moi, tout était évident, j'avais tout compris tout de suite - les papiers, les grades etc. Il s'est dit : "Celle-là, elle est faite pour ». Et après, je n'ai plus jamais quitté la photo. J'avais 15 ans.

En plus, son affaire a décollé, car je n'avais pas mon pareil pour expliquer aux gens comment marchait un appareil photo, le rapport entre l'ouverture du diaphragme et les vitesses, etc. De bouche à oreille, les gens disaient : « Va à Sucy-en-Brie, il y a une petite chez Froissard, elle va t'expliquer comment ça marche... » Il y a avait la queue. J'étais très patiente, et je ne lâchais pas les gens jusqu'au moment où j'étais sûre qu'ils avaient vraiment compris. Donc, lui, il était très content de m'avoir chez lui. J'étais infatigable. J'avais une énergie que j'ai gardée très longtemps. J’y suis restée trois ans.

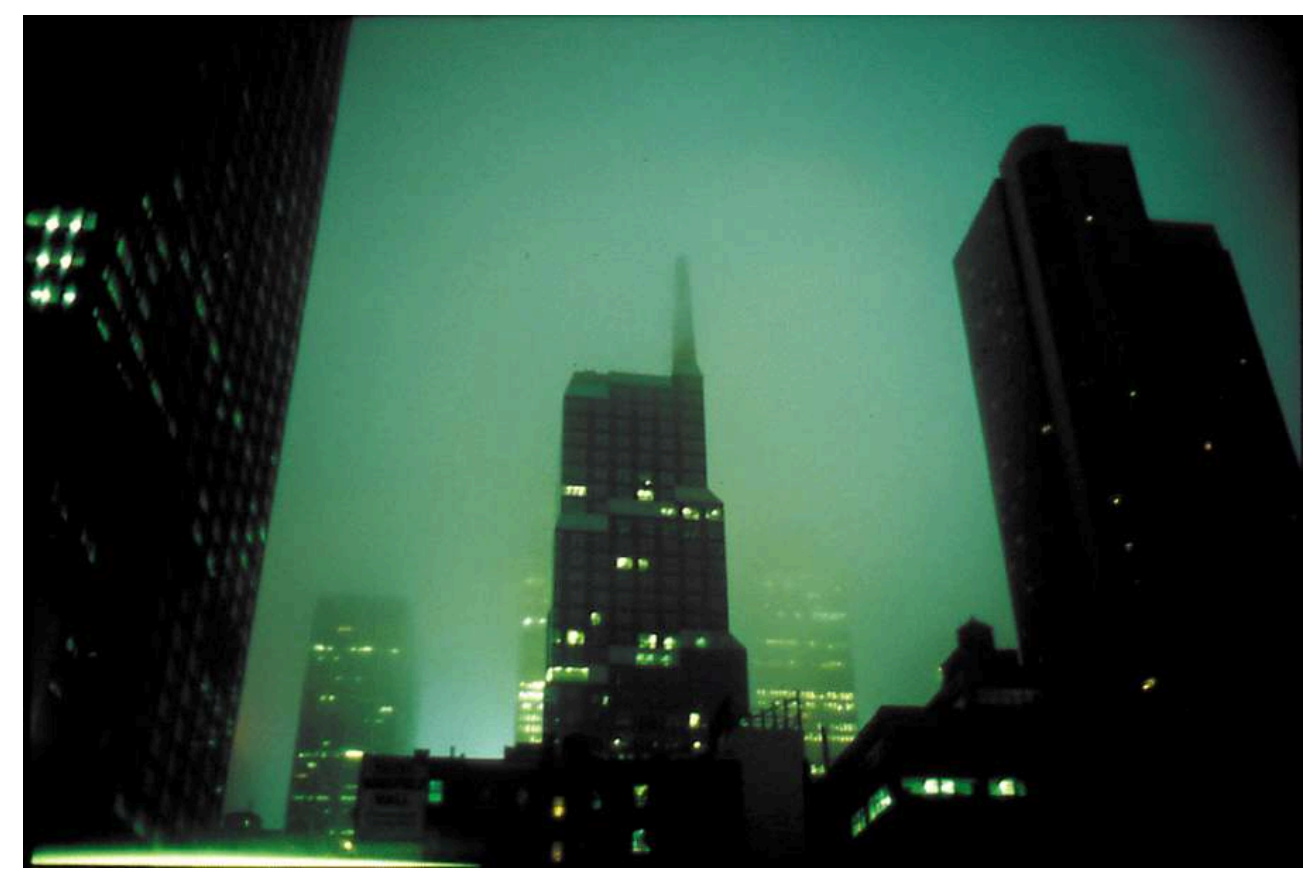

New York, Sans titre [n.d] (102-3)

MA : Votre premier séjour à New York était bien plus tardif?

DM : Oui, c'était bien plus tard. J'ai été à New York en 1994 faire des photos de vitrines de Noël pour un magazine que je ne connaissais pas. C'était quelque chose d'assez technique. La ville m'a tellement plu. L'énergie, c'est une réalité à New York. Je n'y suis pas restée longtemps, trois jours. Je m'étais jurée que je-ne-sais-par-quelmiracle j'y reviendrai pour travailler pour moi. 


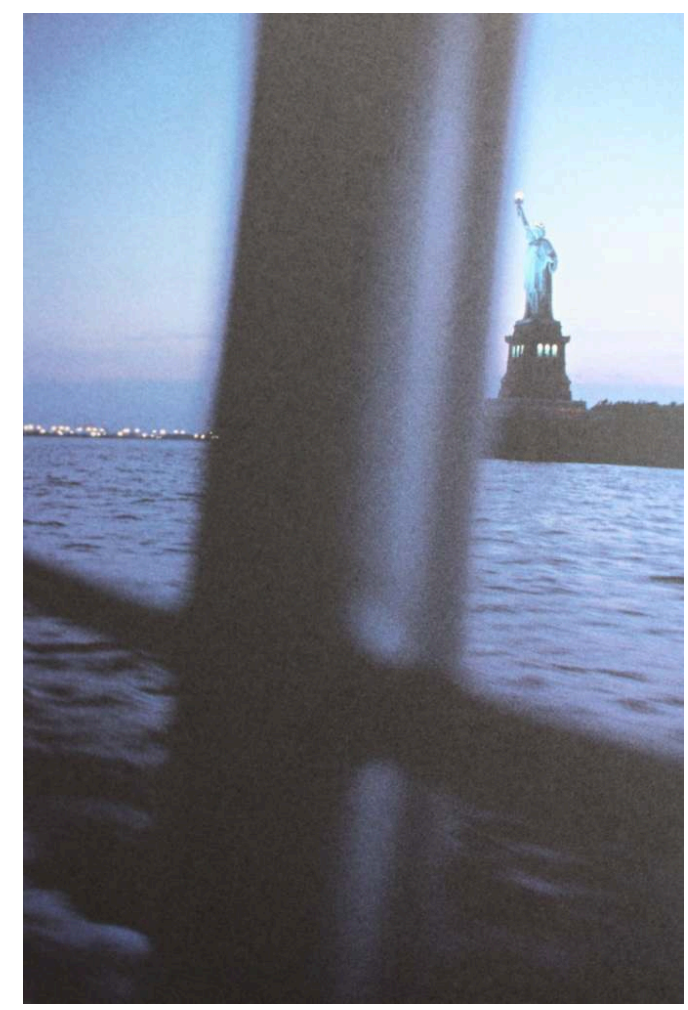

New York, Sans titre [n.d] (188)

Six ans après, j'avais deux amies qui habitaient New York et un jour, je leur dis : « Oh j'adorerais travailler à New York, mais entre le voyage, les bobines, et l'hôtel, c'était trop financièrement pour moi. » Les deux m'ont dit : «Dolores, tu aurais pu nous le dire avant. Bien sûr tu viens dormir chez nous. On se débrouille, on met un lit de camp. » Une semaine après, j'étais à New York. J'ai fait des allers-retours à New York pendant 7 ans. Dès que j'avais trois sous pour me payer l'avion, les bobines et de quoi payer ma nourriture. Je ne restais pas longtemps, car j'avais un compagnon à l'époque qui détestait que je parte, alors on avait un deal, je ne partais pas plus d'une semaine. J'arrivais à New York, je prenais le bus d'abord, puis le métro, je posais mon sac chez mes amies et j'étais dehors. Je commençais dès la première journée, tout de suite. Je marchais, pour voir quelque chose qui me touche et que j'aie envie de photographier. J'étais tellement excitée d'être à New York, je n'étais pas fatiguée. Le décalage horaire, dans ce sens-là, me convenait parfaitement.

Quand j'ai dit à mes amis à Paris que je voulais faire un livre sur New York, tout le monde me disait : «Tu es folle, n'importe quoi, il y en a déjà 100 000. » Je répondais : " Je vais faire un livre sur ma vision à moi de New York. » Donc, voilà, j'ai fait mon livre sur New York.

\section{MA : Comment le qualifieriez-vous, ce New York à vous?}

DM : Très maratéen. [pause] Pour beaucoup de photos, on ne se rend pas compte qu'on est à New York. Un peu comme mes photos à Paris. C'est ma façon de voir. Il y a effectivement quelques photos d'immeubles qui peuvent faire penser que c'est New York. Mais ce qui m'intéresse, avant tout, c'est la solitude des gens dans les villes. Il y a un monde fou dans le métro ou ailleurs, et tout d'un coup, il y a une personne que j'arrive à isoler, dans sa bulle, et cela me touche énormément. C'est assez simple. 


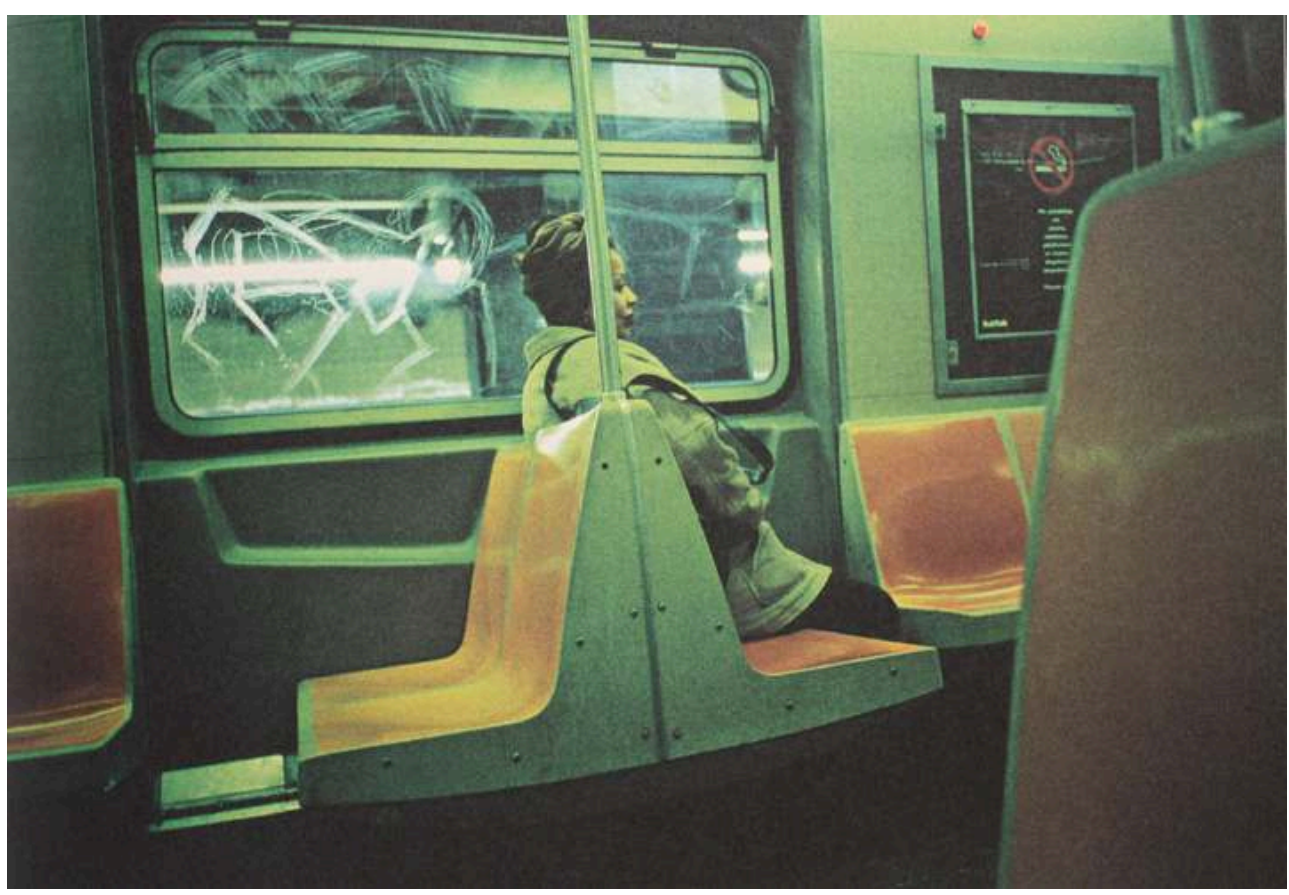

New York, Sans titre [n.d] (89)

Je n'ai jamais été embêtée par les gens que je photographiais. La plupart du temps, ils ne s'en aperçoivent pas. Alors, un jour, il y a un grand type noir, je ne sais pas pourquoi, il était beau ou il était habillé d'une certaine façon. Bizarrement, il me parle en français. "Là, tu as fait une photo." Je lui ai dit: «Non, j'ai pas fait de photos. » En fait, j'avais fait une photo. Il me tutoie, donc je le tutoie, et avec l'instinct de survie, je lui dis : « Tu as vu le flash partir? » « Non. » « Alors tu vois ? » C'est drôle d'avoir dit cela, car je ne fais jamais, mais alors jamais de flash, même pour les photos de commande ou les portraits. Bref, il est parti. C'est la seule fois en sept ans où quelqu'un m'a dit quelque chose. Mais en général, je marche, je ne m'arrête pas. Je fais cela très vite. Et en plus, je suis habillée très simplement. Mon Leica est sous la veste, je le sors au moment de prendre la photo. Je ne fais pas «pro» du tout. J'avance incognito. Les gens ne me voient pas.

\section{MA : C'est une réelle compétence.}

DM : Je fais les réglages avant. Je ne fais aucune retouche, aucun recadrage, aucun changement de couleur, je ne garde que les photos qui restituent l'émotion que j'ai eue. S'il n'y a pas cette émotion, je les jette. 


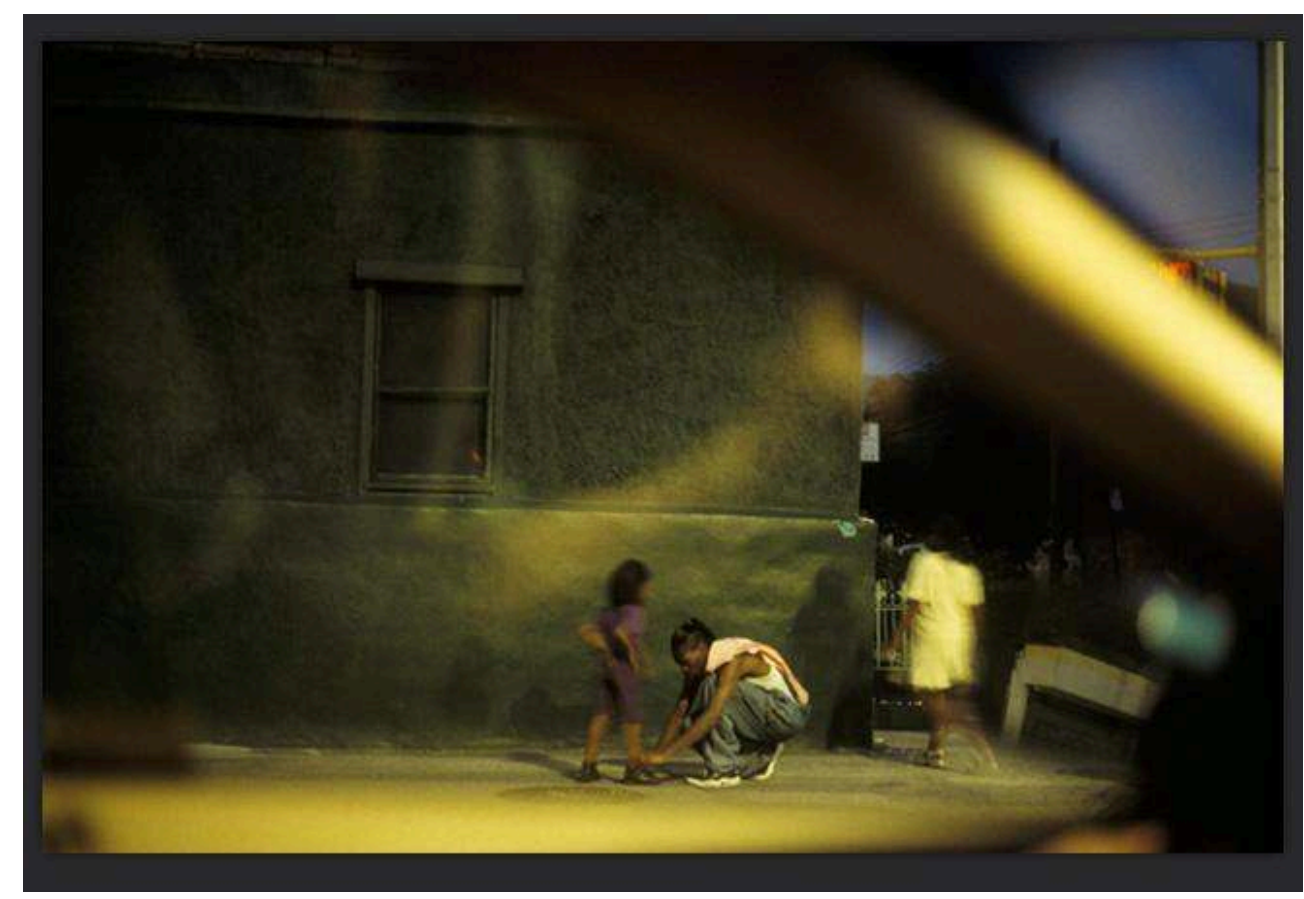

New York, Sans titre [n.d] (42-3)

\section{MA : Pourquoi ne faites-vous pas de recadrages?}

DM : Pour moi, la photo se fait à la prise de vue. Une photo recadrée, je le vois, il y a un décalage, ça me dérange. Il faut que j'arrive à cadrer du premier coup. Quelquefois, il y a des photos que j'ai jetées, car j'avais mal visé ou quelqu'un qui est entré dans le champ. Dans les années 60 , on avait une espèce d'éthique, comme Cartier-Bresson, qui disait lui aussi que la photo se faisait à la prise de vue. Il faut que ce soit beau tout de suite. S'il faut recadrer, pour moi, mais pour moi seulement, c'est une photo loupée.

MA : Vous vous tenez toujours à une même distance des gens. C'est exprès ? Vous ne vous approchez pas. Pas de gros plans. Se tenir à cette distance, c'est pour ne pas être intrusive?

DM : En général, je travaille au $50 \mathrm{~mm}$ ou au $35 \mathrm{~mm}$. Je photographie ce qui me touche, et ce qui me touche, c'est ce que je vois, donc la distance est celle de ma vision. Je ne vais pas me balader avec un téléobjectif.

MA : New York, est-ce une ville que vous avez ressentie différemment? Vous vouliez aller à New York. Or vous dites que vous y avez fait des photos comme à Paris.

DM : New York, c'est plus diversifié que Paris, on change d'ambiance à chaque pâté de maison. Cela me plaisait beaucoup, de ne pas savoir à quoi m'attendre. Je marchais, $\mathrm{du}$ matin jusqu'au soir. Je prenais le bus. Si je voyais une scène qui m'attirait, je descendais à l'arrêt suivant, je revenais à pied sur ce que j'avais vu. C'était avant l'attentat, les gens étaient ouverts, moins peureux que les parisiens. Quand ils voyaient que j'étais perdue, les gens instinctivement me demandaient si j'avais besoin d'aide.

Pendant ces sept ans, j'ai fait deux expositions à New York à la Witkin Gallery, et puis j'ai exposé chez Aperture. Les Américains aimaient bien mon travail. La Witkin Gallery a fermé deux ou trois ans après. 
MA : Quand vous étiez à New York, aviez-vous l'impression que le fait d'être étrangère vous aidait?

DM : Oui, c'était une découverte pour moi, tout était nouveau. Le Nouveau Monde. Tout m'intéressait. Je suis arrivée à faire trois ou quatre voyages par an pendant sept ans. C'est pour cela que je suis triste de n'avoir jamais vu la neige à New York. Je suis hystérique quand il y a de la neige. C'est tellement beau, ce recouvrement.

Je veux voir ce que je suis capable de faire ailleurs qu'à Paris. En Avignon, je vais commencer mes photos quand il y aura des ciels un peu plus gris, maintenant que je n'ai plus ma canne. Je voudrais faire un travail du Palais des Papes sous la neige. Je prie pour qu'il neige. Je me vois sortir dans le froid en pleine nuit. Sinon, il y a trop de monde. Ici dès qu'il fait beau, tout le monde est en short. Toutes ces tables dehors et tout cela, cela ne m'intéresse pas.

MA : Ce qui me frappe dans vos photographies, c'est le sentiment d'absence, les personnes en retrait. Ces personnes, vous les montrez, mais elles ne sont pas vraiment là.

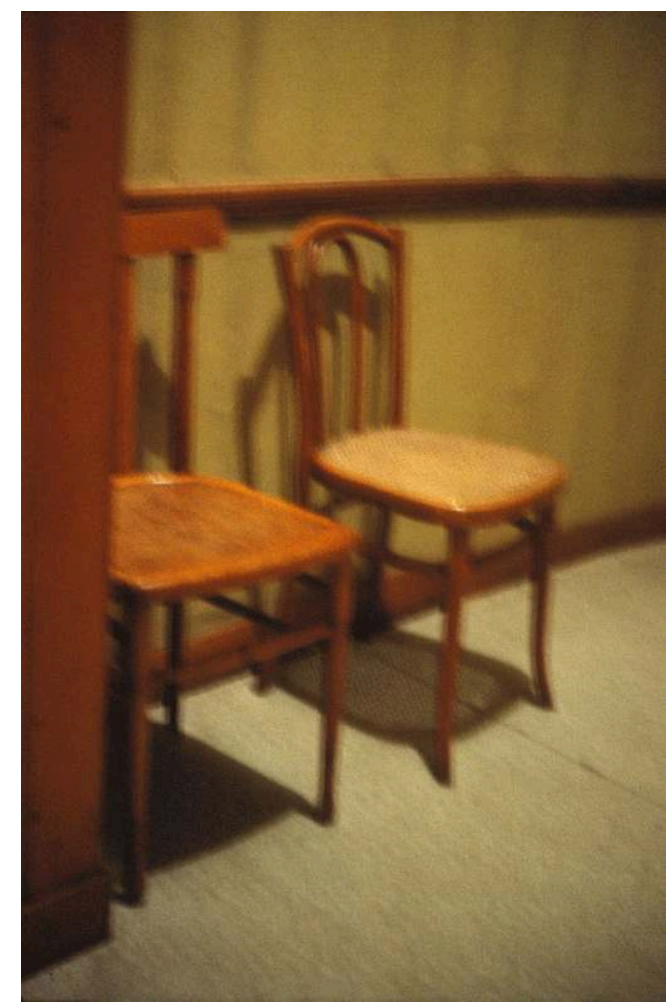

Normandie, Sans titre [n.d] (page de couverture de Passage)

DM : Quand j'étais petite, j'étais à moitié autiste. Quand j'étais à la récréation, j'étais repliée, extrêmement timide. Quand on est trois, je m'efface devant la conversation des deux autres. Depuis qu'on a fait un trou dans ma tête, je suis un peu plus bavarde.

MA : On saisit la gravité de vos photos, mais celles-ci conservent malgré tout une sorte de grâce; vous auriez pu opter pour des images beaucoup plus dures. On comprend la déréliction, l'impression d'abandon des gens que vous photographiez, lesquels sont isolés, comme dans une espèce de stase feutrée.

DM : En fait, j'ai le syndrome de l'abandon et en plus le complexe de ne pas avoir été à l'école. Je suis très complexée. Et entièrement autodidacte. 
MA : Il y a peut-être un bonheur dans votre malheur de ne pas avoir été à l'école longtemps : votre photo n'aurait peut-être pas eu cette poésie. Vous auriez reproduit votre douleur de manière peut-être plus académique.

DM : Peut-être, mais j'aurais vraiment aimé aller à l'école, pour savoir et découvrir des choses que j'ignore encore aujourd'hui.

MA : Vos photos sont souvent nocturnes, ou si elles ne sont pas nocturnes, ce sont vos couleurs qui sont crépusculaires.

DM : Un jour, j'étais envoyée en Corse, pour faire des photos de nuit. C'était une commande. En voyant la lumière de Corse, je me suis dit: "Qu'est-ce que je suis capable de faire au soleil ? ", d'où Sirocco ${ }^{1}$. J'ai vu la mer pour la première fois à 40 ans. Un jour, j'ai eu envie de voir ce dont j'étais capable. Je me suis dit : « Un jour il faudra que j'aille dans les pays du soleil pour voir ce que je peux faire. »

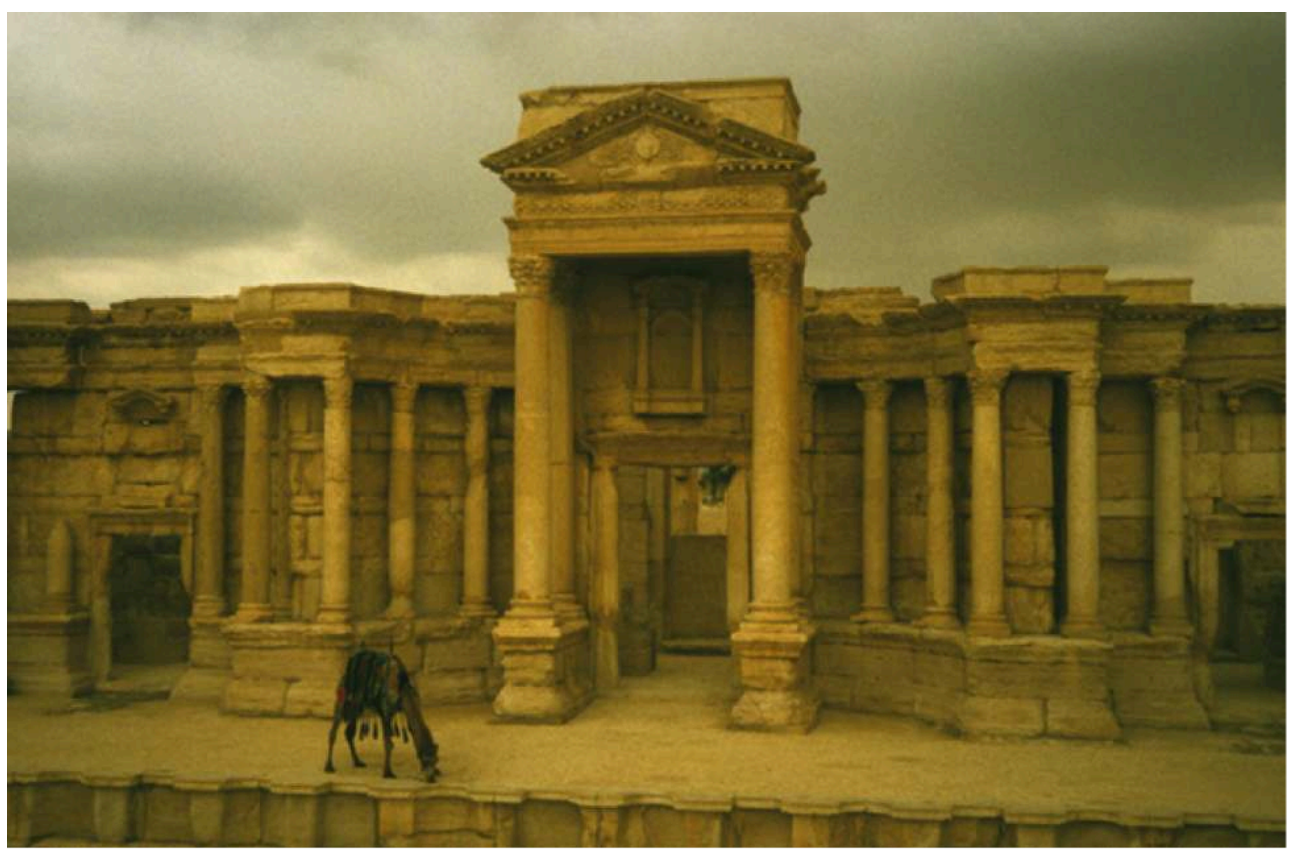

Palmyr, Sans titre [n.d], exposition Sirrocco

MA: Mais dans Sirocco, on ne voit guère le soleil. Les photos restent sourdes et grises, entre chien et loup. Les couleurs sont aussi en retrait.

DM : Cela ne me viendrait pas à l'idée de faire des photos à midi. Je préfère la lumière atténuée, ou à l'heure bleue, ou le matin très tôt. Ou avec un ciel orageux ! 


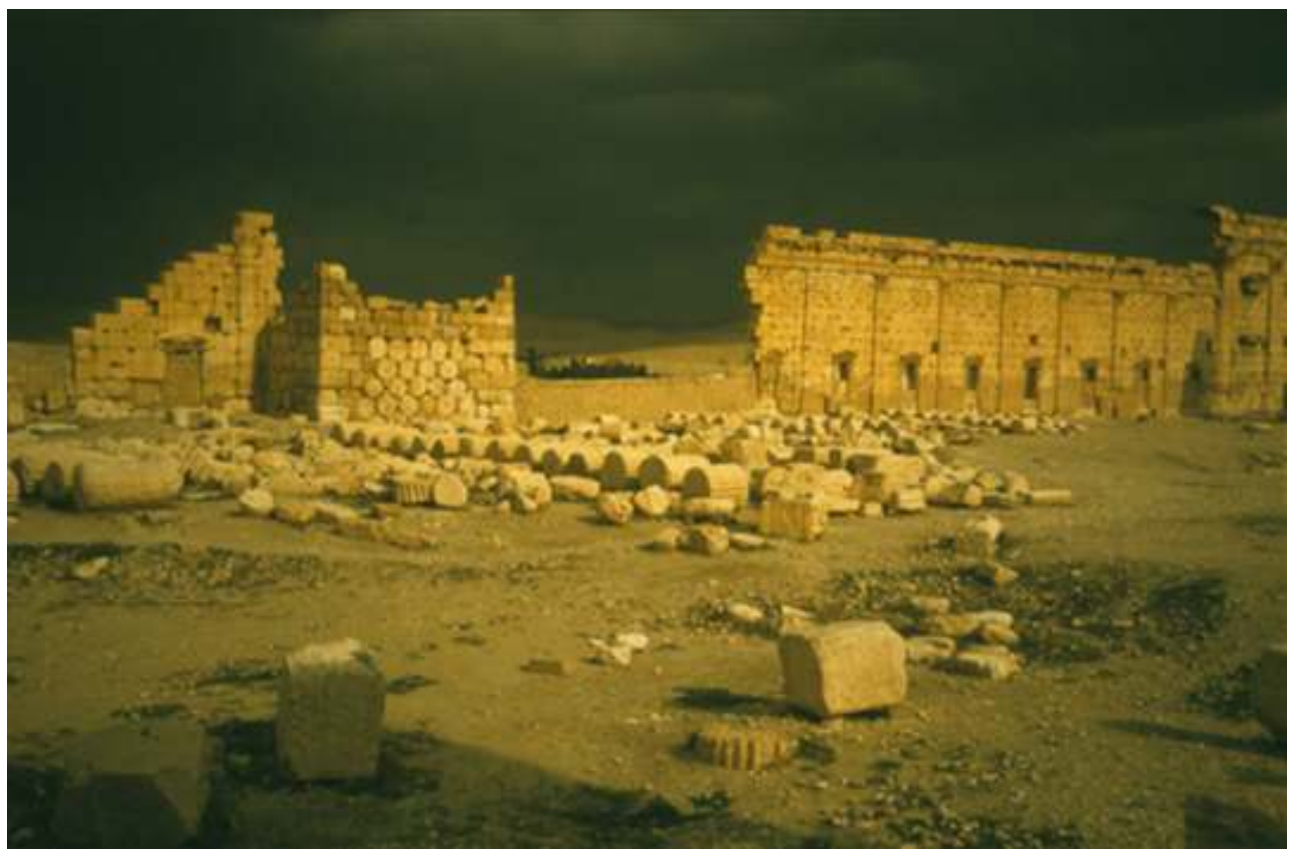

Palmyr, Sans titre [n.d], exposition Sirrocco

MA : Comment savez-vous que vous devez photographier telle ou telle scène ?

$\mathrm{DM}$ : Par rapport à ce que je ressens. Je ne suis pas reporter, je ne veux rien démontrer, rien prouver. Juste trouver une émotion, qui me fait penser à moi quand j'étais petite. Quand je vois quelque chose, je me raconte des histoires. Des souvenirs plus ou moins fabriqués. Par exemple, quand j'ai pris des oiseaux, pour moi, c'était des éclats d'obus. Je suis très sensible aux bruits de guerre.

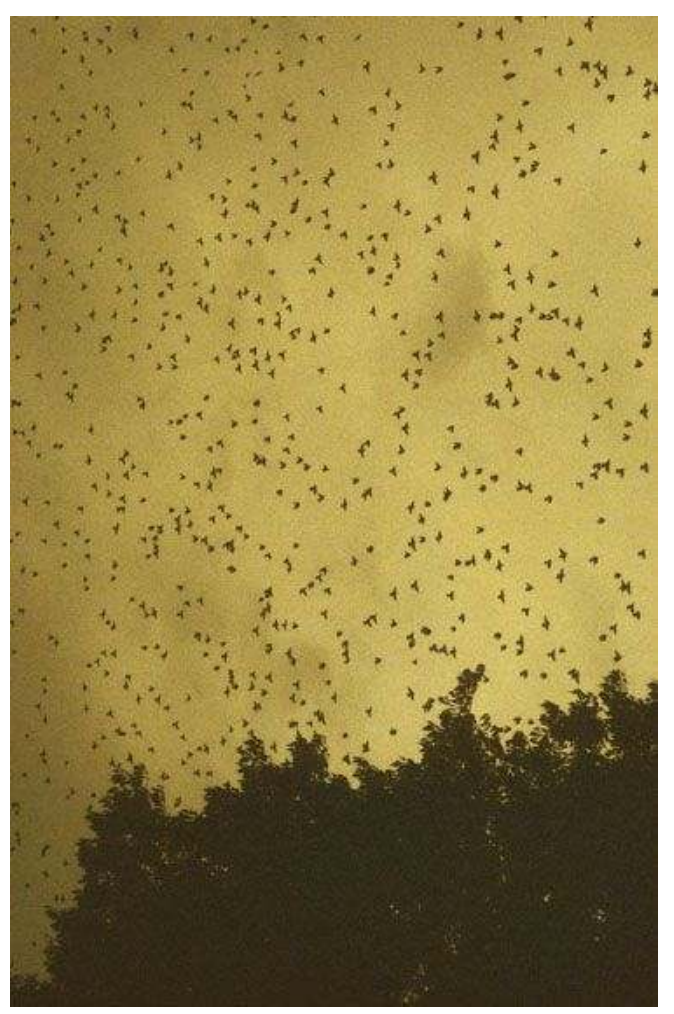

Marseille, Sans titre [n.d] 
MA: Vous avez connu une petite partie de la guerre. Et pourtant, ce qui frappe, c'est l'actualité du style de vos photos, même si elles sont nostalgiques, et d'une certaine façon, intemporelles. Depuis l'âge de 15 ans, vous avez l'impression que votre style a évolué?

DM : Non.

MA : Vous reconnaissez-vous dans un courant actuel ?

DM : Non, en dehors de tout. Les gens de la photo me connaissent, je crois. Mais je n'ai jamais fait d'exposition dans un lieu officiel. J'ai mis cela sur le compte du fait que je ne suis jamais allée à l'école, que je ne sais pas trop bien parler. Je ne suis pas capable de faire un discours, et cela ne leur convient peut-être pas. Je peux raconter ma vie, mais je n'ai pas de discours sur mon travail.

MA: Vous connaissiez Sarah Moon?

DM : J'ai tiré des photos de Sarah Moon quand je travaillais pour le magazine pour lequel j'étais laborantine.

MA : On peut trouver des similitudes entre vos images : les couleurs, l'aspect suranné.

DM : Dans le livre sur New York, il y a deux photos faites par Sarah Moon et moi à quelques années près. Ni elle ni moi, je pense, n'avions vu les images de l'autre. Une exposition chez Agnès B s'intitulait «Leurre ». Agnès B avait demandé la photo du cowboy. Cette photo avait pu être faite à New York. Sarah Moon avait acheté cette photo à Agnès B.

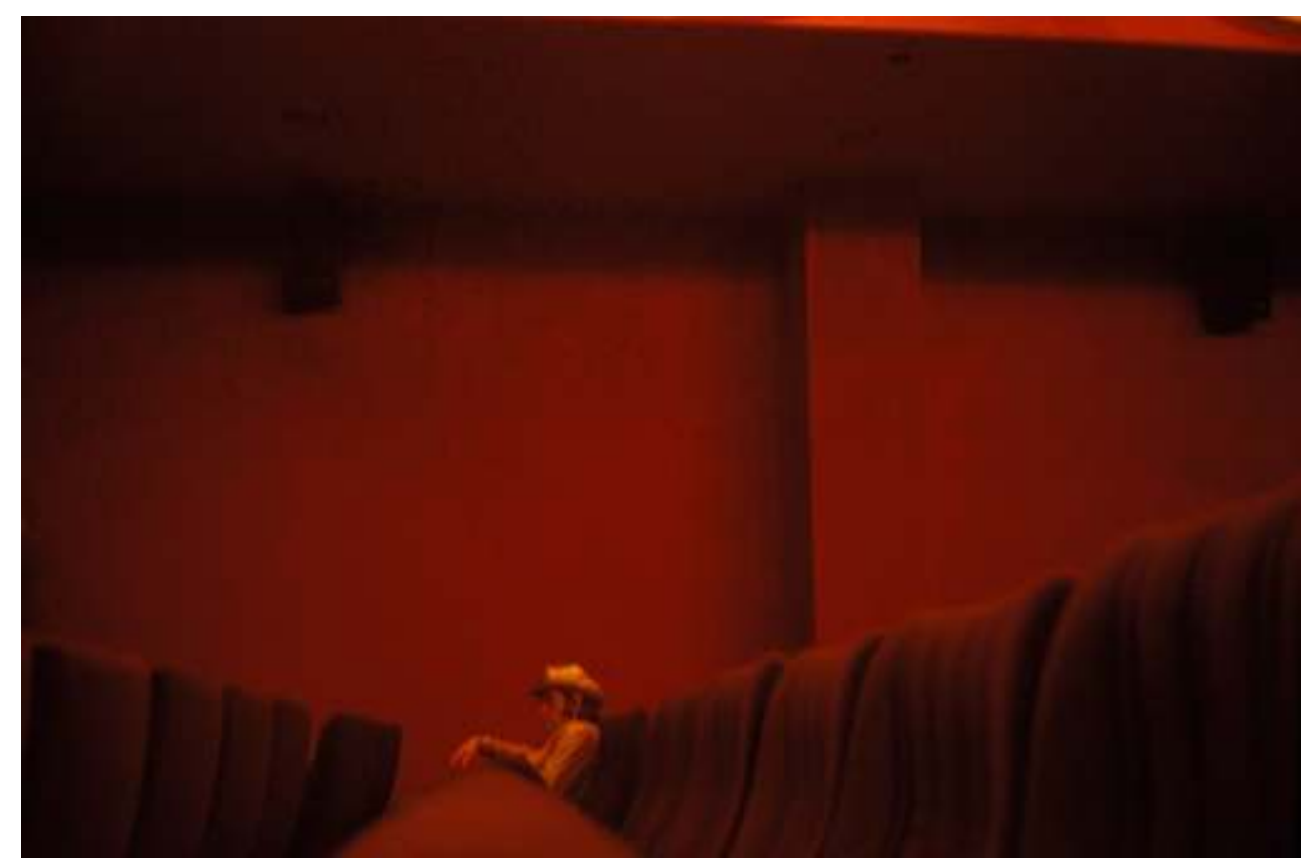

Paris, Sans titre [n.d] 


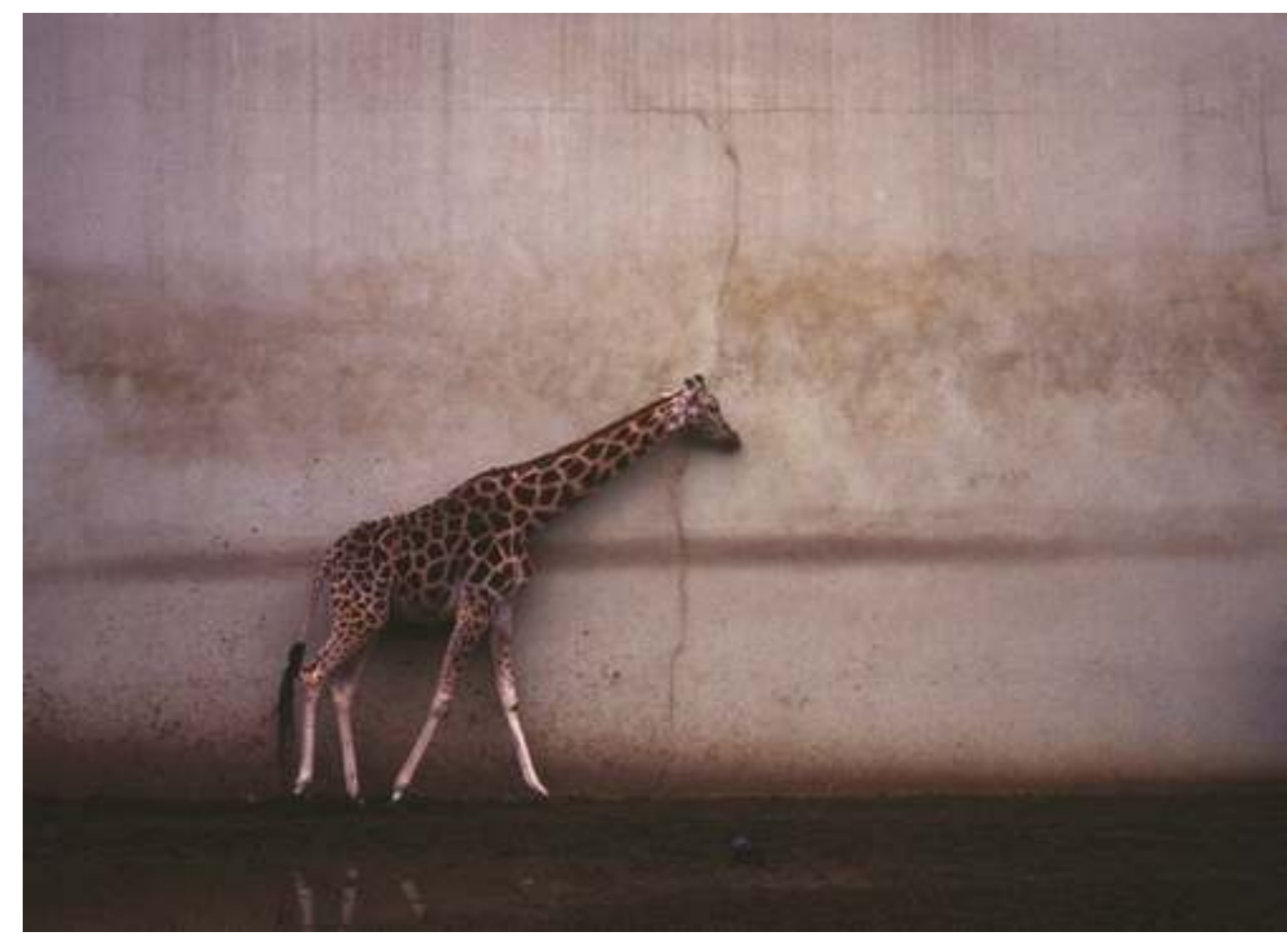

Barcelone, Sans titre [n.d]

MA : Votre photographie de girafe aurait pu être faite par Sarah Moon.

DM : Oui. Je voudrais faire un jour un livre sur l'enfermement des animaux dans les zoos $^{2}$.

\section{MA : Une autre sorte d'orphelinat?}

DM : Oui. Ici, j'ai deux photos, déjà : la girafe et le singe. Mes photos sont dans un entrepôt. J'ai fait mon décor avec les photos que j'avais chez moi.

[Dolores Marat me raconte un terrible accident qu'elle a subi il y a quelques années.]

Depuis les deux ans et demi que j'ai quitté Paris, ce n'est pas facile pour moi. A l'hôpital, je voulais prendre mon appareil photo. Mais tout le monde me disait que j'allais me le faire voler. Il faut dire que j'étais très mal en point.

MA : Quel dommage ! À l'hôpital, il y a de quoi photographier. Et puis faire de la photo, cela redonne de l'énergie.

DM : Bien sûr, mais le souci, c'est que je ne pouvais plus utiliser mes mains. Quand j'étais en rééducation, je n'avais pas très chaud, donc je mettais un petit gilet. Je mettais exactement 20 minutes par bouton, montre en main. Les infirmières, quand elles rentraient dans la chambre, voulaient m'aider. Mais je voulais le faire moi-même pour réhabituer mes mains. Mes mains ne répondent plus. Je suis incapable d'ouvrir une bouteille minérale. Je n'ai plus de poigne. Je suis incapable de porter un litre et demi avec ma main gauche. Cela, c'est lié à l'AVC que j'ai fait à la suite de l'accident. Les infirmières m'ont dit: «C'est vous qui avez raison, on peine à vous voir, mais c'est bien d'avoir cette force de vouloir vous en sortir.» [...]. Mais je galère pas mal aujourd'hui. Comme je vis seule, je n'ai plus d'aide. Quand mes amis viennent, ils peuvent m'aider, mais ils ne sont pas là tous les jours. Mon fils et ma fille sont à St Malo. Je me débrouille. Quand on est photographe, on est bricoleur et plein de ressources. 
MA : Quand on est photographe, on est en quête de quelque chose, qu'il faut trouver.

DM : Surtout que moi-même, au niveau professionnel, j'étais toute seule dans mon labo et dans le studio du magazine; il fallait que je trouve les solutions. J'ai appris sur le tas. Je n'hésiterai pas à recommencer, encore et encore.

MA : Comme vous êtes autodidacte, c'est comme cela que vous concevez l'apprentissage. C'est l'avantage de ne pas avoir été à l'école : vous avez une opiniâtreté que n'ont pas ceux qui ont eu l'apprentissage facile et qui se découragent au moindre obstacle.

DM : J'ai toujours été habituée à me former toute seule, même petite.

MA: Certaines scènes sont des sortes d'anecdotes. Prenez par exemple la photographie des quatre enfants autour d'une flaque.

DM : Là, c'est aussi du pur hasard, comme toutes mes photos. C'était à Brooklyn. J'étais là, sur les conseils d'une amie. Je suis comme une enfant à New York. Un des enfants a jeté un caillou et les quatre enfants ont regardé les ronds dans l'eau. J'étais sur le chemin, je n'ai eu le temps de ne faire qu'une photo et puis j'ai continué ma route. C'est des cadeaux qu'il faut savoir saisir.

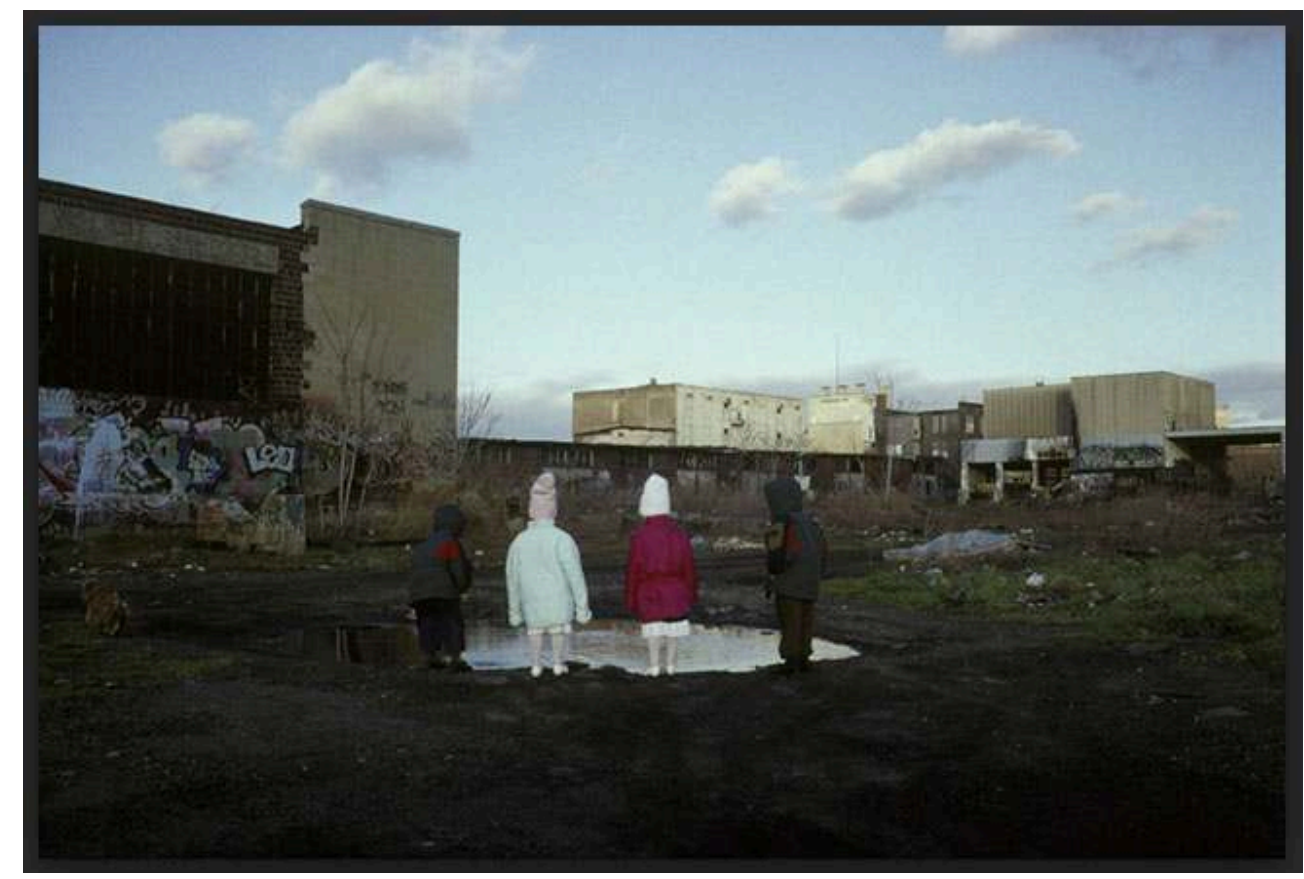

New York, Sans titre [n.d] (105)

MA : Ce n'est pas donné à tout le monde de pouvoir les repérer.

DM : Mais, moi, je suis tendue par la photo $24 \mathrm{~h} / 24$. Même quand je dors, je fais des rêves, des cauchemars au sujet de mes appareils photos. Je rêve, par exemple, que j'ai un trou dans l'appareil.

MA : Dans ce cas, c'est l'appareil qui a un AVC et non plus vous-même.

DM : Exactement, oui. La première fois qu'Hermès m'envoya faire des photos, c'était pour l'ouverture d'un magasin à Los Angeles. D'un seul coup, je regarde dans mon appareil photo. Rien. Comme si je n'avais plus de pile. Instinctivement je le retourne, et qu'est-ce que je vois? Un trou. Les piles n'étaient plus là, mais surtout il manquait la petite vis. Les piles sont sur le devant. Des petites piles pas plus grosses que l'ongle de mon petit doigt. Je me suis dit: «Parmi tous ces gens-là, Dolores, retrouve ta vis $»^{3}$. Comme quoi je suis tenace. Il y avait 5000 personnes. La rue était bloquée sur 
4-5 kilomètres. Et puis, il y avait une table immense car il y avait un buffet. J'ai commencé à trouver une pile, puis j'ai retrouvé la deuxième pile un peu loin. Puis un peu plus loin encore, j'ai retrouvé la vis. C'est incroyable que personne n'ait shooté dedans. Quelqu'un m'avait dit : « De toute façon, toi, tu as un ange gardien. » Pendant longtemps, j'ai cru que j'avais un ange gardien. Peut-être que j'en ai un, encore.

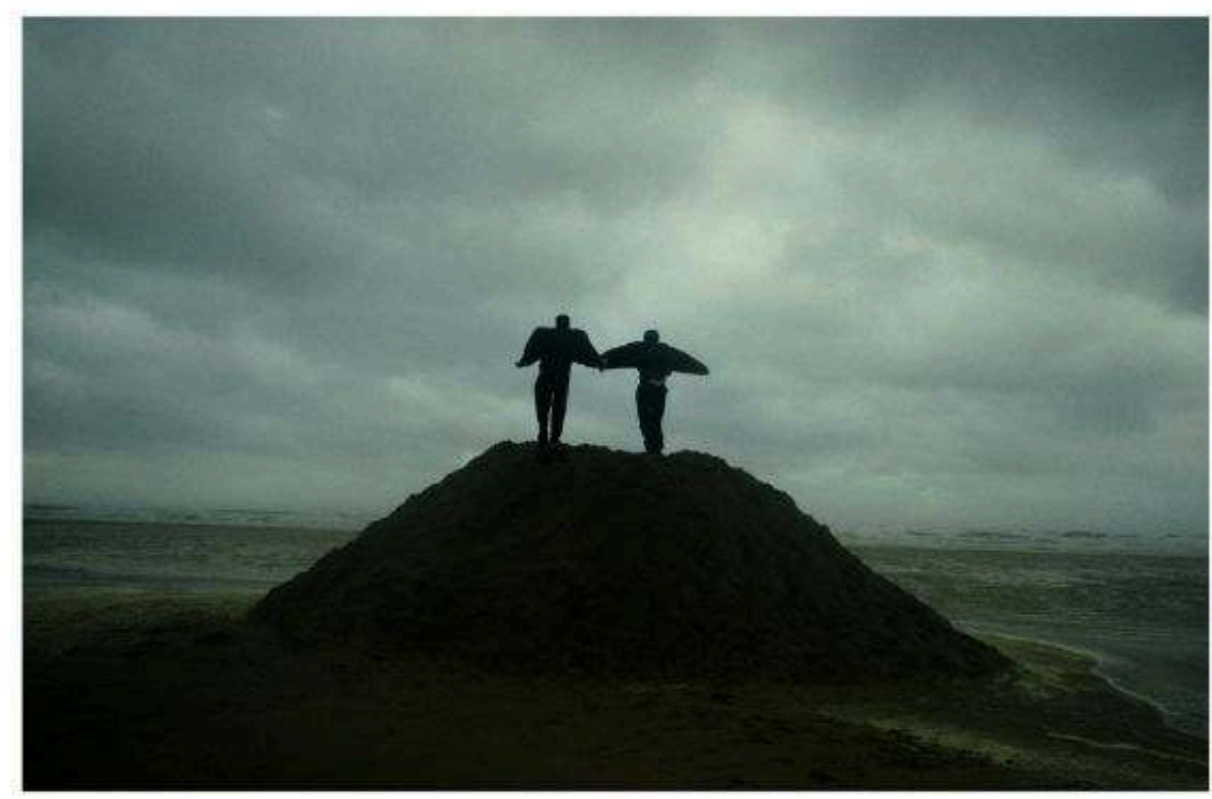

Dieppe, Sans titre [n.d]
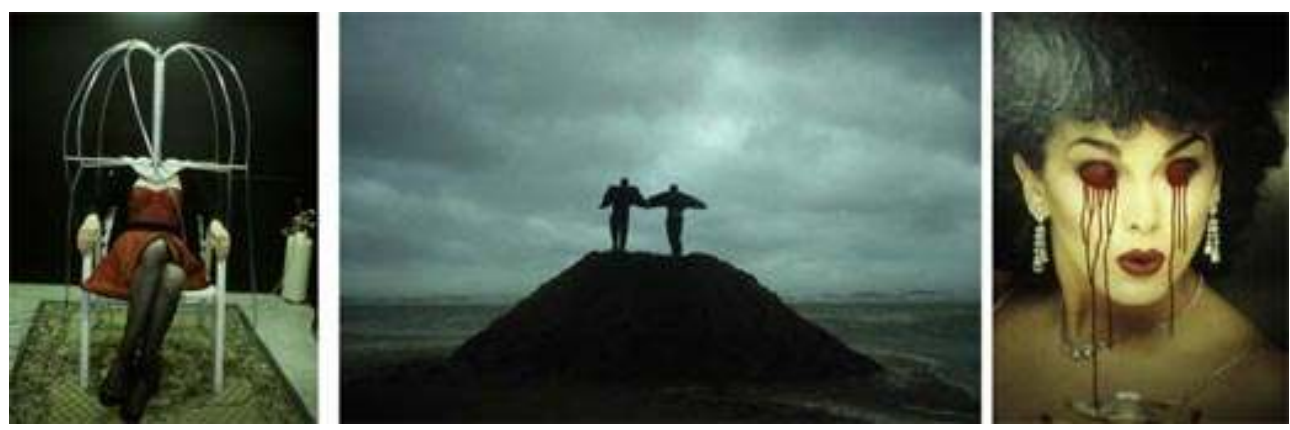

MA : Si les miracles sont un leitmotiv de votre vie, alors quand cela vous arrive d'être au fond du trou, vous pouvez garder une lueur d'espoir.

DM : Oui, c'est cela. Je n'abandonne pas. Je m'accroche. 


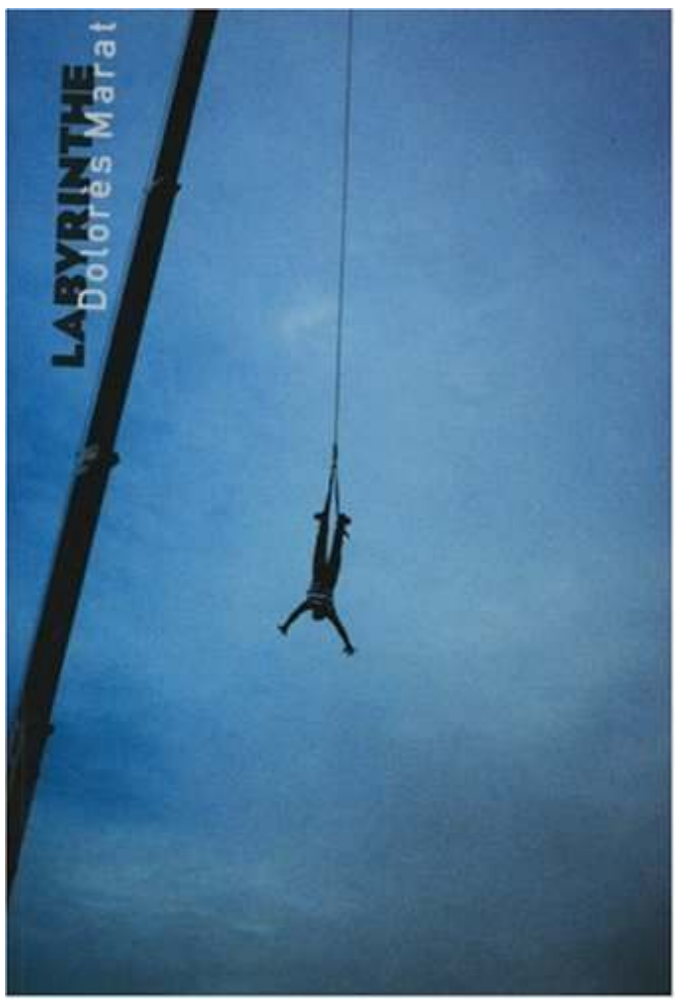

Paris, Sans titre [n.d] (page de couverture de Labyrinthe)

2 Dolores Marat vit désormais en Avignon. Voilà son site : http://www.doloresmarat.fr/ photographe/accueil.php

\section{NOTES}

1. Titre d'une exposition qui a eu lieu à Marseille du 14 octobre au 3 décembre 2010, à Toulouse du 9 avril au 3 mai 2013, et à Paris du 16 mai au 22 juin 2013.

2. Sur le site de Dolores Marat, toute une série porte sur l'enfermement des animaux.

3. Notons au passage la paronymie entre « vis » et « vie » (note de Muriel Adrien).

\section{INDEX}

Thèmes : Trans'Arts 